\title{
Expression of gene for Dioscorea batatas tuber lectin 1 in transgenic tobacco confers resistance to green-peach aphid
}

\author{
Tetsuya Kato ${ }^{1}$, Masatoshi Hori ${ }^{2}$, Tomohisa Ogawa ${ }^{3}$, Koji Muramoto ${ }^{3}$, \\ Kinya Toriyama ${ }^{1, *}$ \\ ${ }^{1}$ Laboratory of Environmental Biotechnology, Graduate School of Agricultural Science, Tohoku University, Sendai, \\ Miyagi 981-8555, Japan; ${ }^{2}$ Laboratory of Insect Science and Bioregulation, Graduate School of Agricultural Science, \\ Tohoku University, Sendai, Miyagi 981-8555, Japan; ${ }^{3}$ Laboratory of Functional Biomolecules, Graduate School of Life \\ Sciences, Tohoku University, Sendai, Miyagi 981-8555, Japan \\ *E-mail: torikin@bios.tohoku.ac.jp Tel: +81-22-717-8830 Fax: +81-22-717-8834
}

Received December 8, 2009; accepted January 18, 2010 (Edited by K. Yazaki)

\begin{abstract}
Dioscorea batatas tuber lectin 1 (DB1) is a storage protein isolated from yam tuber, and is shown to be a mannose-binding lectin. It has $58 \%$ amino-acid identity to insecticidal snowdrop bulb lectin GNA. In this study, we demonstrated that $\geq 1 \mathrm{mg} \mathrm{ml}^{-1} \mathrm{DB} 1$ in an artificial diet significantly decreased the survival and fecundity of green peach aphid, Myzus persicaeca. We produced transgenic tobacco plants expressing cDNA of DB1 under the control of Cauliflower mosaic virus $35 \mathrm{~S}$ promoter (35S-DB1) or phloem-specific promoter of rice sucrose synthase-1 gene (RSs1-DB1), and evaluate the degree of aphid resistance in whole plant bioassays. The number of survival aphids was reduced to $60 \%$ in transgenic lines with 35S-DB1 and RSs1-DB1, which accumulated DB1 at a level of $1.8 \%$ and $0.25 \%$, respectively, of total soluble protein. Our results indicate that DB1 can be used to enhance resistance to sap-sucking insects in transgenic crops.
\end{abstract}

Key words: Aphid, Dioscorea batatas tuber lectin 1, insect resistance, transgenic tobacco.

Genetically modified (GM) crops are now cultivated worldwide, and insect-resistant crops accounted for 18\% of GM-cultivated area in 2007 according to a report of the International Service for the Acquisition of AgriBiotech Applications (ISAAA Brief 37-2007; http:// www.isaaa.org/resources/Publications/briefs/37/). All insect-resistant GM crops contain a $C r y$ gene encoding Bt toxin of Bacillus thuringiensis. The Cry gene has been shown to be useful for controlling various chewing insects such as lepidopteran and coleopteran larvae. Bt transgenic crops, however, are known to be susceptible to sap-sucking insects such as aphids and planthoppers (Rao et al. 1998). In contrast, snowdrop bulb lectin (Galanthus nivalis agglutinin; GNA), which was isolated from an ornamental monocotyledonous plant, has been successfully used to enhance resistance to sap-sucking insects in transgenic tobacco (Hilder et al. 1995), maize (Wang et al. 2005) and rice (Nagadhara et al. 2003; 2004; Rao et al. 1998). GNA belongs to the mannosebinding lectin family. Mannose-binding proteins, in general, are known to bind to mannose-containing glycoproteins in the mid-guts of insects, and to inhibit growth and development (Sauvion et al. 1996).

DB1 has been isolated from yam tuber, Dioscorea batatas Decne., as a storage protein. DB1 is a monnose- binding lectin $(23 \mathrm{kDa})$ consisting of identical $12-\mathrm{kDa}$ subunits. It has $58 \%$ amino-acid identity to snowdrop lectin GNA and is, thus, classified in the GNA-related lectin family (Gaidamashvili et al. 2004). The insecticidal properties of DB1 have been reported against moth larvae (Helicoverpa armigera). The rate of adults emerging from pupae has been reduced to $33 \%$, when fed on $0.1 \mathrm{mg} \mathrm{ml}^{-1}$ DB1 in an artificial diet (Ohizumi et al. 2009). A gene for DB1 is, therefore, expected to be useful for the production of insect-resistant crops.

In this study, we first examined the insecticidal activity of DB1 protein against aphids using an artificial diet assay. Then we produced transgenic tobacco expressing DB1 cDNA under the control of Cauliflower mosaic virus (CaMV) 35S promoter or rice sucrose synthase-1 (RSs1) promoter. The RSs1 promoter has been reported to be a phloem-specific promoter and is thought to be more effective on sap-sucking insects and minimize any potential undesirable accumulation of the protein in other parts of plants (Rao et al. 1998). The insecticidal activity of DB1 against aphids was demonstrated in the whole plant assay of the transgenic plants.

This article can be found at http://www.jspcmb.jp/ 


\section{Materials and methods}

\section{Artificial diet bioassays}

Green peach aphid (Myzus persicae) was obtained form JT (Japan Tobacco Inc. Japan). Aphids were maintained on mature plants of Nicotiana tabacum cv. Petit Havana SR1 in a controlled environment growth chamber, $25 \pm 1^{\circ} \mathrm{C}, 16 \mathrm{~L} / 8 \mathrm{D}$.

The artificial diet bioassays for aphids was carried out as described by Powell et al. (1993) using an artificial diet for aphids (Dadd and Mitter 1966). DB1 was incorporated at 2, 1 and $0.1 \mathrm{mg} \mathrm{ml}^{-1}$ in the artificial diet. Controls were set as treatment with feed using an artificial diet containing $0 \mathrm{mg} \mathrm{ml}^{-1}$ DB1 or $1 \mathrm{mg} \mathrm{ml}^{-1} \mathrm{BSA}$, and treatment with no diet (no diet controls). Ten adult apterous aphids were transferred from the host plant to glass petri-dishes. Each petri-dish was sealed with a stretched Parafilm (Pechiney Plastic Packaging Inc., USA), an $500 \mu \mathrm{l}$ artificial diet was placed on top of the stretched Parafilm, and then the artificial diet was covered with another stretched Parafilm. The diet and Parafilm were changed daily. The bioassays were conducted in an environmental growth chamber $\left(25 \pm 1^{\circ} \mathrm{C}, 16 \mathrm{~L} / 8 \mathrm{D}\right)$, and the number of surviving adults and newborn nymphs was counted with four sets of replication. A statistical analysis was carried out using the Excel Statistics software.

\section{Transformation of tobacco}

The nucleotide sequence for a DB1 isoform, DB1 (Leu86), under the accession no. AB178475 in DDBJ was revealed to be lacking a part of $5^{\prime}$ signal sequence. PCR for 5' RACE was carried out to obtain full length cDNA using yam tuber mRNA (Ohizumi et al. 2009) and the Marathon cDNA Amplification Kit (BD Bioscience Clontech). The nucleotide sequence of full length open reading frame (ORF) of DB1 cDNA was deposited to DDBJ (accession no. AB513659). It encodes 172 aminoacid protein with a predicted targeting-signal sequence to endoplasmic reticulum.

The cDNA covering full-length ORF was PCR cloned into pGEM T-vectors (Promega, Madison, USA) using KOD+ polymerase (Toyobo, Osaka, Japan) and primers XB/NDB1 F 5'-TCTAGAGGATCCATGGCTAACCCAGGAGCA-3' (Xba I and Bam HI sites are underlined) and S/DB1 R 5'-GAGCTCTCACTTGTTGACGACC-3' (Sac I site is underlined). The PCR products were digested with $\mathrm{Bam} \mathrm{HI} / \mathrm{Sac}$ I and inserted into Bam HI/Sac I sites of pBE2113 (Mitsuhara et al. 1996), which contained CaMV 35S promoter with doubled enhancer and omega sequence of tobacco mosaic virus for enhanced expression. The resulting construct, 35S-DB1, was used for strong and constitutive expression of the DB1 gene. For phloem-specific expression of DB1, the rice sucrose synthase-1 (RSs1) promoter (accession no. AJ401233) was employed as described (Shi et al. 1994). The promoter sequence $(3 \mathrm{~kb})$ was amplified from rice cv. Taichung 65 using $\mathrm{KOD}+$ polymerase and specific primers Sal/RSs1 F 5' - GTCGACCTTTCGTGACTTGTTTTCGC-3' (Sal I site is underlined) and Bam/ RSs1_R 5'-GGATCCTAGCTTGGCAGCCAT-3' (Bam HI site is underlined), and was subcloned into pGEM-T vector. Then RSs1 promoter was inserted into Sal I/Bam H I sites of pBI101H, which contained the hygromycin resistance cassette (Ariizumi et al. 2002). The GUS gene in pBI101H was replaced by DB1 cDNA at Bam HI/Sac I sites. The resulting construct was named RSs1-DB1. These constructs were transferred into Agrobacterium tumefaciens strain EHA105 (Hood et al. 1993). Transformation of tobacco (Nicotiana tabacam L. Petit Havana SR1) was carried out using a standard leaf-disk method. The transformants were selected on shootinducing medium containing $50 \mathrm{mg}^{-1}$ kanamycin and $1 \mathrm{mg}^{-1}$ meropen (Dainippon Sumitomo Pharma Co., Ltd., Osaka, Japan).

\section{Western blot analysis}

The total soluble protein (TSP) was extracted from leaves of $\mathrm{T}_{0}$ and $\mathrm{T}_{1}$ transgenic tobacco in $50 \mathrm{mM}$ Tris- $\mathrm{HCl}(\mathrm{pH} \mathrm{7.0)}$. The concentration of extracted protein was determined using the Bio-Rad protein assay kit. Approximately $\sim 10 \mu \mathrm{g}$ of TSP was separated on $12 \%$ SDS-PAGE and transferred onto PVDF membrane $(0.45 \mu \mathrm{m}$, Millipore, USA). The membrane was reacted with anti-DB1 polyclonal antiserum at $1: 10,000$ dilution (Ohizumi et al. 2009) and anti-rabbit IgG Alkaline phosphatase conjugate (Promega, USA) as secondary antibody at $1: 5,000$ dilution. Bound secondary antibody was detected using nitro blue tetrazolium and 5-bromo-4-chloro-3-indolyl phosphate (Wako Chemicals, Tokyo, Japan). The DB1 concentration in TSP was determined by comparing the intensity of bands with those of series of known amounts of purified DB1.

\section{Whole plant bioassays}

Transgenic tobacco plants $\left(\mathrm{T}_{1}\right)$ with accumulated $\mathrm{DB} 1$ and untransformed wild-type tobacco (WT) were grown in 5-inch pots in a controlled environmental growth room $\left(25 \pm 1^{\circ} \mathrm{C}\right.$, $16 \mathrm{~L} / 8 \mathrm{D})$. When plants were about $20 \mathrm{~cm}$ tall, each plant was transferred into a $30 \times 30 \times 40 \mathrm{~cm}$ plastic case and infested with 5 adult apteraous aphids. The numbers of aphids on each plant were counted daily. Four plants were tested for each transgenic line.

\section{Results}

\section{Effect of DB1 on green peach aphid in artificial diet bioassays}

The insecticidal activity of DB1 against green peach aphid was tested at 2, 1 and $0.1 \mathrm{mg} \mathrm{ml}^{-1} \mathrm{DB} 1$ in the artificial diet. The number of survivors was counted at one to seven days of feeding (Figure 1). The number of survivors was greatly reduced to almost zero after 3 days in the no diet control, while it was slightly reduced when administrated with $1 \mathrm{mg} \mathrm{ml}^{-1} \mathrm{BSA}$ and $0 \mathrm{mg} \mathrm{ml}^{-1} \mathrm{DB} 1$. Addition of $1 \mathrm{mg} \mathrm{ml}^{-1}$ and $2 \mathrm{mg} \mathrm{ml}^{-1} \mathrm{DB} 1$ significantly $(\mathrm{P}<0.01)$ reduced the number of survivors in comparison with treatments containing $1 \mathrm{mg} \mathrm{ml}^{-1} \mathrm{BSA}$ and $0 \mathrm{mg} \mathrm{ml}^{-1} \mathrm{DB} 1$. No aphids survived after 7 days of feeding with $2 \mathrm{mg} \mathrm{ml}^{-1} \mathrm{DB} 1$.

Corrected mortality was calculated at the 3rd day when the number of survivors in the no diet control reached zero as follows: (number of survivors in $0 \%$ 


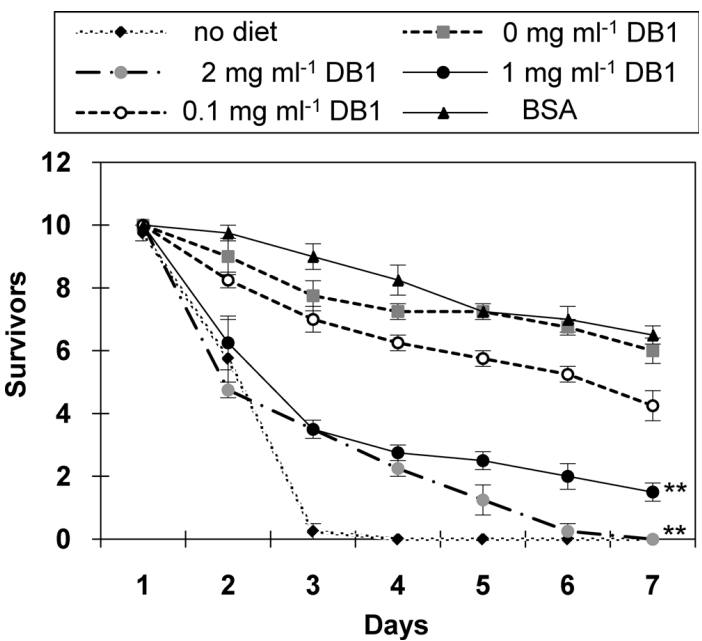

Figure 1. The number of survivors of aphids in artificial diet bioassay $(n=4)$. DB1 was incorporated at 2,1 and $0.1 \mathrm{mg} \mathrm{ml}^{-1}$ in the artificial diet. Controls were set as treatment with feed using an artificial diet containing $0 \mathrm{mg} \mathrm{ml}^{-1}$ DB1 or $1 \mathrm{mg} \mathrm{ml}^{-1} \mathrm{BSA}$, and treatment with no diet. The survival rate was statistically analyzed using the Kaplan-Meier method and significance of differences was analyzed by the log-rank test. **implies a significant difference between control $\left(0 \mathrm{mg} \mathrm{ml}^{-1} \mathrm{DB} 1\right)$ and each treatment at $\mathrm{P}<0.01$.

DB1 condition-number of survivors in the condition of designated concentration of DB1)/(Number of survivors in $0 \% \mathrm{DB} 1$ condition) $\times 100$ (Abbot1925). Corrected mortality was $70 \%$ in $2 \mathrm{mg} \mathrm{ml}^{-1}$ DB1 condition, $62 \%$ in $1 \mathrm{mg} \mathrm{ml}^{-1}$ DB1 and $13 \%$ in $0.1 \mathrm{mg} \mathrm{ml}^{-1} \mathrm{DB} 1$. These results indicated that DB1 has insecticidal activity in a concentration-dependent manner.

The number of nymphs was also counted every day. The number of nymphs per number of living aphids was around 0.92 and 0.83 when fed with $0 \mathrm{mg} \mathrm{ml}^{-1} \mathrm{DB} 1$ diet and $1 \mathrm{mg} \mathrm{ml}^{-1}$ BSA-containing diet for 4 days respectively, whereas it was reduced to 0.48 in the case of $0.1 \mathrm{mg} \mathrm{m}^{-1}$ DB1 condition, and approximately 0.2 in cases of $1 \mathrm{mg} \mathrm{ml}^{-1}$ and $2 \mathrm{mg} \mathrm{ml}^{-1}$ DB1 (Figure 2). The difference was significant $(\mathrm{P}<0.05)$ at the 5 th day. These results indicate that DB1 decreased the survival and fecundity of green peach aphid.

\section{Transgenic tobacco accumulating $D B 1$}

We produced several lines of transgenic tobacco plants with 35S-DB1 or RSs1-DB1. The accumulated DB1 in leaves was detected using western blot analysis. An approximately $12-\mathrm{kd}$ band corresponding to mature DB1 monomer was detected at the same position as that of standard DB1 purified form yam tuber, indicating the proper processing of $16-\mathrm{kd}$ DB1 premature protein in transgenic tobacco (Figure 3). In the case of transgenic lines with 35S-DB1, DB1 accumulated at a level of 2.4\% of total soluble protein in plant no. $35 \mathrm{~S}-1,2.0 \%$ in no. $35 \mathrm{~S}-6$ and $1.4 \%$ in no. $35 \mathrm{~S}-7$. The progeny of no. $35 \mathrm{~S}-1$ (nine plants of $\mathrm{T}_{1}$ generation with $35 \mathrm{~S}-\mathrm{DB} 1$ ) also contained DB1 at almost the same level with an average

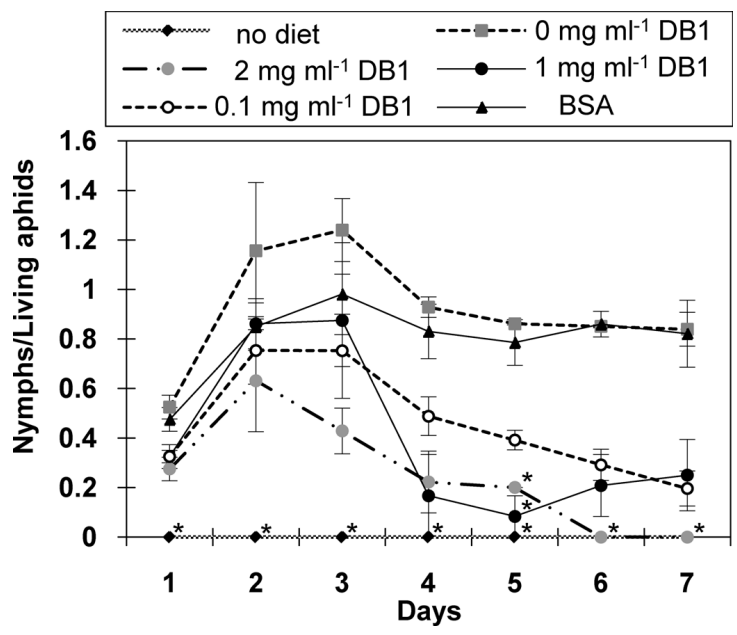

Figure 2. The number of nymphs per number of living aphids in artificial diet bioassay $(n=5)$. DB1 was incorporated at 2, 1 and $0.1 \mathrm{mg} \mathrm{ml}^{-1}$ in the artificial diet. Controls were set as treatment with feed using an artificial diet containing $0 \mathrm{mg} \mathrm{ml}^{-1} \mathrm{DB} 1$ or $1 \mathrm{mg} \mathrm{ml}^{-1}$ BSA, and treatment with no diet. *implies a significant difference between control $\left(0 \mathrm{mg} \mathrm{ml}^{-1} \mathrm{DB} 1\right)$ and each treatment at $\mathrm{P}<0.05$ by Steel's test after Kruskal-Wallis test.

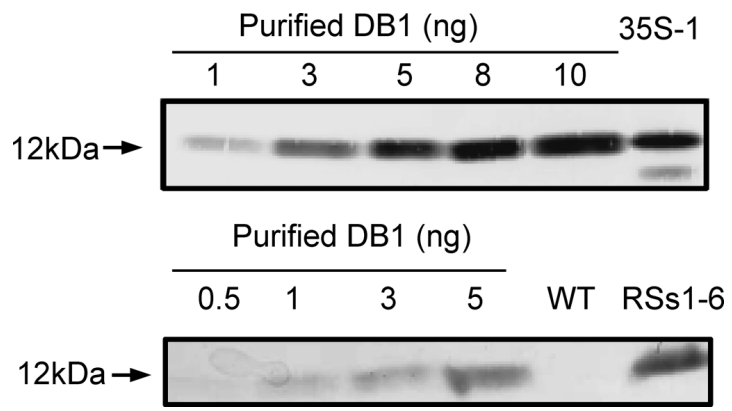

Figure 3. Western blot analysis of DB1 in leaves of transgenic tobacco with 35S-DB1 (35S-1) and RSs1-DB1 (RSs1-6) and nontransgenic tobacco (WT). The DB1 concentration in transgenic tobacco was determined by comparing the intensity of bands with those of series of known amounts of purified DB1 (0.5 to $10 \mathrm{ng}$ ). Five $\mu \mathrm{g}$ of total soluble protein were loaded for each transgenic tobacco and WT.

of $1.8 \%$.

In the case of transgenic lines with RSs1-DB1, DB1 accumulated at a level of $0.1 \%$ of total soluble protein in plant nos. RSs1-2 and RSs1-3, and 0.25\% in no. RSs1-6. The progeny of no. RSs1-6 (nine plants of $\mathrm{T}_{1}$ generation with RSs1-DB1) also contained DB1 at the same level of $0.25 \%$ on average.

\section{Effect of DB1 on green peach aphid in whole plant bioassays}

Four $\mathrm{T}_{1}$ plants of the each transgenic line, no. 35S-1 and no. RSs1-6, in which accumulation of DB1 was confirmed, were used for evaluation of the degree of aphid resistance in whole plant bioassays. Transgenic line no. 35S-13 accumulating no detectable DB1 and non-transformed WT were used as a negative control. The number of aphids per plant was counted up to 14 
days of bioassays (Figure 4). At the 14th day, the number of aphids on average was $481 \pm 21$ in plant no. $35 \mathrm{~S}-1$, and $492 \pm 14$ in plant no. RSs1-6, while it was $786 \pm 72$ in WT and $755 \pm 18$ in 35S-13 (negative control). The number of aphids per plant was significantly $(\mathrm{P}<0.05)$ reduced to $60 \%$ in $\mathrm{DB} 1$-accumulating tobacco compared to non-accumulating tobacco. No differences were found in the number of aphids between the 35S-DB1 line and the RSs1-DB1 line. This result clearly demonstrated the transgenic tobacco expressing DB1 enhanced resistance to green peach aphid.

\section{Discussion}

An LD50 for DB1 in artificial diet was estimated to be $0.67 \mathrm{mg} \mathrm{ml}^{-1}$ at the 4th day and $0.27 \mathrm{mg} \mathrm{ml}^{-1}$ at the 7 th day based on Probit analysis. Concentrations more than $1 \mathrm{mg} \mathrm{ml}^{-1}$ caused significant reduction of mortality and fecundity. The corrected mortality of $1 \mathrm{mg} \mathrm{ml}^{-1} \mathrm{DB} 1$ was $62 \%$. The toxicity of lectin against green peach aphid has also been reported for snowdrop lectin GNA (Hilder et al. 1995). The reported corrected mortality of $1 \mathrm{mg} \mathrm{ml}^{-1}$ GNA was $33 \%$. Thus the toxicity of DB1 was similar to that of GNA. GNA has been reported to show insecticidal activity against other sap-sucking insects. The corrected mortality of $1 \mathrm{mg} \mathrm{ml}^{-1}$ GNA against brown planthopper (Nilaparvta lugens) and green rice planthopper (Nephotterix cinciteps) was reported to be $79 \%$ and $87 \%$, respectively (Powell et al. 1993). DB1 is expected to have entomotoxic effects against such planthoppers.

We employed CaMV35S promoter and RSs1 promoter to direct DB1 expression in tobacco. Transgenic tobacco accumulating GNA up to $2.5 \%$ total soluble protein, which was expressed under the control of CaMV35S promoter, has been shown to decrease the number of living green peach aphids to $56 \%$ in leaf disk bioassays (Hilder et al. 1995). The insecticidal effect of DB1 on aphid in transgenic tobacco is similar to that of the previous report of GNA. Similar results have been also reported for garlic lectin (Allium sativum agglutinin from leaf; ASAL), which shares a $48 \%$ amino- acid identity with DB1. Expression of ASAL under the control of the phloem-specific promoter Asusl from Arabidopsis thaliana has been reported to protect tobacco plants against the tobacco aphid (Myzus nicotianae) with a reduction of $40 \%$ of reproduction capacity (Sadeghi et al. 2007). GNA and ASAL have been shown to confer substantial resistance to brown planthopper, green rice planthopper, and whitebacked planthopper, in terms of increased insect mortality, retarded development and decreasing fecundity, when expressed in transgenic rice (Nagadhara et al. 2003; 2004; Saha et al. 2006; Yarasi et al. 2008). Recently, virus resistance has been also reported in transgenic rice expressing ASAL under the

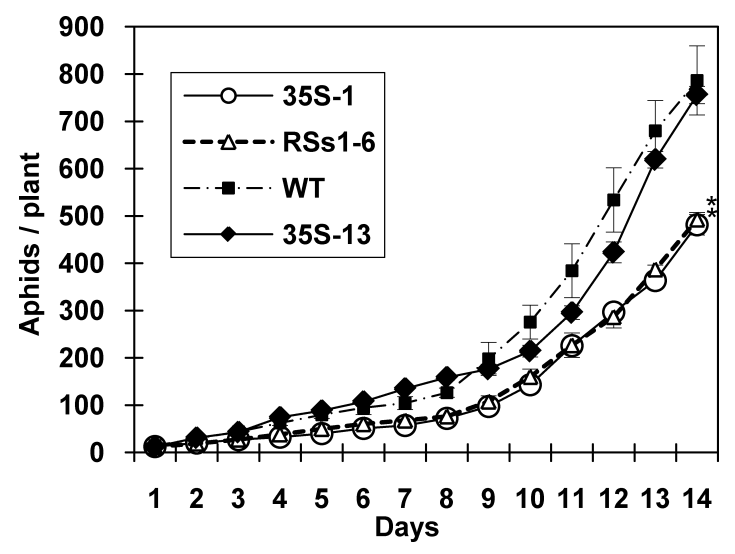

Figure 4. The number of aphids per transgenic tobacco plant with 35S-DB1 (35S-1), RSs1-DB1 (RSs1-6) and WT $(n=4)$. DB1 accumulated at a level of $1.8 \%$ of total soluble protein in the $35 \mathrm{~S}-1$ line and $0.25 \%$ in the RSs $1-6$ line. No. $35 \mathrm{~S}-13$ is a negative control accumulating no detectable DB1. *implies a significant difference between WT and each transgenic plant at $\mathrm{P}<0.05$ by Steel's test after Kruskal-Wallis test.

control of RSs1 promoter (Saha et al. 2006). We are now investigating the resistance against planthoppers and rice tungro virus in transgenic rice plants expressing DB1.

RSs1 promoter has also been shown to direct phloemspecific expression of beta-glucuronidase and GNA in transgenic tobacco (Shi et al. 1994). RSs1 promoter has also been used to drive GNA in transgenic rice (Nagadhara et al. 2003; 2004) and maize (Wang et al. 2005), and to drive garlic lectin, ASAL, in transgenic rice (Saha et al. 2006). RSs1 promoter has the advantage of maximizing expression of the insecticidal protein at the site of attack by sap-sucking insects, while minimizing it elsewhere in plants. Our current study demonstrated that the level of aphid resistance is the same between the RSs1-DB1 and 35S-DB1 lines, although the amount of DB1 per soluble protein extracted from a whole leaf of the RSs1-DB1 line was approximately one-seventh of that in the 35S-DB1 line, suggesting that the amount of DB1 in phloem might be almost identical. Our results confirmed the effectiveness of RSs1 promoter.

DB1 was isolated from yam tubers of Japanese cultivar, which are generally eaten as raw tubers without boiling. DB1, therefore, is considered to be least nonharmful to human beings. Taking our current study into consideration, the DB1 gene would be useful for producing safe GM crops with resistance to sap-sucking insects.

\section{Acknowledgements}

This study was supported by Dean's Fund for Frontier Research on Agricultural Sciences, Tohoku University. 


\section{References}

Abbott WS (1925) A method of computing the effectiveness of an insecticide. J Economy Entomol 18: 265-267

Ariizumi T, Kishitani S, Inatsugi R, Nishida I, Murata N, Toriyama $K$ (2002) An increase in unsaturation of fatty acids in phosphatidylglycerol from leaves improves the rates of photosynthesis and growth at low temperatures in transgenic rice seedlings. Plant Cell Physiol 43: 751-758

Dadd RH, Mitter TE (1966) Permanent culture on an aphid on a totally synthetic diet. Experientia 22: 832-833

Ewen SWB, Pusztai A (1999) Effect of diets containing genetically modified potatoes expressing Galanthus nivalis lectin on rat small intestine. Lancet 354: 1353-1354

Gaidamashvili M, Ohizumi Y, Iijima S, Takayama T, Ogawa T, Muramoto K (2004) Characterization of yam tuber storage proteins from Dioscorea batatas exhibiting unique lectin activities. J Biol Chem 279: 26028-26035

Hilder VA, Powell KS, Gatehouse AMR, Gatehouse JA, Gatehouse LN, et al. (1995) Expression of snowdrop lectin in transgenic tobacco plants results in added protection against aphids. Transgenic Res 4: 18-25

Hood E, Gelvin SB, Melchers LS, Hoekema A (1993) New Agrobacterium helper plasmids for gene transfer to plants. Transgenic Res 2: 208-218

Mitsuhara I, Ugaki M, Hirochika H, Ohsimima M, Murakami T, et al. (1996) Efficient promoter cassettes for enhanced expression of foreign genes in dicotyledonous and monocotyledonous plants. Plant Cell Physiol 37: 49-59

Nagadhara D, Ramesh S, Pasalu IC, Rao YK, Krishnaiah NV, et al. (2003) Transgenic indica rice resistant to sap-sucking insects. Plant Biotechnol J 3: 231-240

Nagadhara D, Rameshi S, Pasalu IC, Rao YK, Sarma NP, Reddy VD, Rao KV (2004) Transgenic rice plants expressing the snowdrop lectin genes (GNA) exhibit high-level resistance to the whitebacked planthopper (Sogatella furcifera). Theor Appl Genet 109: 1399-1405

Ohizumi Y, Gaidamashvili M, Ohwada S, Matsuda K, Kominami J, et al. (2009) Mannose-binding lectin from yam (Dioscorea batatas) tubers with insecticidal properties against Helicoverpa armigera (Lepidoptera: Noctuidae) J Agric Food Chem 57: 2896-2902

Powell KS, Gatehouse AMR, Hilder VA, Gatehouse JA (1993) Antimetabolic effects of plant lectins and plant and fungal enzymes on the nymphal stages of two important rice pests, Milaparvata lugens and Nephotettix cinciteps. Entomol Exp Appl 66: 119-126

Rao KV, Rathore KS, Hodges TK, Fu X, Stoger E, et al. (1998) Expression of snowdrop lectin (GNA) in transgenic rice plants confers resistance to rice brown hopper. Plant J 15: 469-477

Sadeghi A, Broeders S, De Greve H, Hernalsteens JP, Peumans WJ, Van Damme EJ, Smagghe G (2007) Expression of garlic leaf lectin under the control of the phloem-specific promoter Asus 1 from Arabidopsis thaliana protects tobacco plants against the tobacco aphid (Myzus nicotianae). Pest Manage Sci 63: 12151223

Saha P, Majumder P, Dutta I, Ray T, Roy SC, Das S (2006) Transgenic rice expressing Allium sativum leaf lectin with enhanced resistance against sap-sucking insect pests. Planta 223: $1329-1343$

Sauvion N, Rahbe Y, Peumans W, Damme V (1996) Effect of GNA and other mannose binding lectins on development and fecundity of the peach-potato aphid Myzus persicae. Entomol Exp Appl 79: 285-293

Shi Y, Wang MB, Power KS, Damme EV, Hilder VA, Gahehouse AMR, Boulter D, Gatehouse JA (1994) Use of the rice sucrose synthase-1 promoter to direct phloem-specific expression of $\beta$ glucuronidase and snowdrop lectin genes in transgenic tobacco plants. J Exp Bot 45: 623-631

Wang Z, Zhang K, Sun X, Tang K, Zhang J (2005) Enhancement of resistance to aphids by introducing the snowdrop lectin gene gna into maize plants. J Biosci 30: 627-638

Yarasi B, Sadumpati V, Immanni CP, Vudem DR, Khareedu VR (2008) Transgenic rice expressing Allium sativum leaf agglutinin (ASAL) exhibits high-level resistance against major sap-sucking pests. BMC Plant Biology 8: 102 